
\title{
25 Research Soure \\ Impact of High-Risk Features for Stage II Adenocarcinoma of the Appendix
}

\section{Mehmet Akce ( $\nabla$ mehmet.akce@emory.edu )}

Emory University https://orcid.org/0000-0003-4747-7722

Katerina Zakka

Emory University https://orcid.org/0000-0003-1498-6424

\section{McKenna Penley}

Emory University

\section{Renjian Jiang}

Emory University

\section{Olatunji B. Alese}

Emory University

Walid L. Shaib

Emory University

Christina Wu

Emory University

Madhusmita Behera

Emory University

Bassel F. El-Rayes

Emory University

\section{Research article}

Keywords: Appendix adenocarcinoma, stage II, high risk, low risk, adjuvant chemotherapy, clinical outcomes, treatment, chemotherapy

Posted Date: May 29th, 2020

DOI: https://doi.org/10.21203/rs.3.rs-31568/v1

License: (c) (i) This work is licensed under a Creative Commons Attribution 4.0 International License. Read Full License

Version of Record: A version of this preprint was published at Journal of Clinical Oncology on February 1st, 2020. See the published version at https://doi.org/10.1200/JC0.2020.38.4_suppl.795. 


\section{Abstract}

Background: Clinico-pathological high-risk features are frequently utilized in adjuvant chemotherapy (AC) decisions in stage II colorectal cancer and their utility in stage II appendiceal adenocarcinoma (AA) is not established. The aim of this study is to determine the impact of high-risk features in clinical outcomes and whether high risk features are predictive of AC benefit in stage II AA.

Methods: Patients with pathological stage II AA between 2010-2015 were identified from the National Cancer Database (NCDB) using ICD-0-3 morphology and topography codes: 8140, 8480 and C18.1. High risk stage II AA was defined as having at least one of the following clinicopathological features: T4 tumor, $<12$ lymph nodes examined, poorly differentiated histology, positive margins, or lymphovascular invasion. Patients with none of these features were defined as low-risk.

Results: A total of 1,040 patients with pathological stage II AA were identified. $51.0 \%$ males, $84.5 \%$ Caucasian; median age 61 (range, 19-90). 46.4\% were determined to have high-risk stage II AA. High-risk status was associated with worse OS compared to low-risk in univariate (HR 1.55; 95\% Cl 1.18-2.02; $\mathrm{p}=0.001)$ and multivariable analyses $(\mathrm{HR} 1.36 ; 95 \% \mathrm{Cl} 1.03-1.79 ; \mathrm{p}=0.028)$. High-risk stage II AA patients had significantly worse 5 -year OS compared to low-risk patients $(67.1 \%$ vs. $74.5 \%, p=0.0013)$. AC was administered in $34.4 \%(n=166)$ of high-risk patients and in $36.5 \%(n=203)$ of low-risk patients. Among high-risk patients, AC was not associated with better OS in univariate (HR 0.86; 95\% Cl 0.59-1.26; $p=0.448)$ and multivariable analyses (HR $1.35 ; 95 \% \mathrm{Cl} 0.90-2.04 ; \mathrm{p}=0.151)$ compared to no AC. Similarly, among low-risk patients, AC was not associated with better OS in univariate (HR 0.92; 95\% Cl 0.60-1.39; $\mathrm{p}=0.679)$ and multivariable analyses (HR 1.27; $95 \% \mathrm{Cl} 0.81-2.02 ; \mathrm{p}=0.299)$ compared to no AC. For highrisk patients, 5 -year OS was $68.3 \%$ in patients that received AC vs. $66.5 \%$ in patients that did not $(p=0.722)$. For low-risk patients, 5 -year OS was $74.0 \%$ in patients that received AC vs. $76.3 \%$ in patients that did not $(p=0.813)$.

Conclusion: High-risk stage II AA patients had significantly worse 5-year OS compared to low-risk patients. AC did not improve survival regardless of high-risk features in stage II AA.

\section{Background}

Appendiceal neoplasms are rare and have various histologic types such as adenocarcinoma, neuroendocrine tumors, goblet/ex-goblet cell carcinoid, mixed neuroendocrine-non-neuroendocrine neoplasm (MiNEN) $(1,2)$. Appendiceal adenocarcinoma (AA) constitutes around $0.5 \%$ of all gastrointestinal tract neoplasms $(3,4)$. AA is frequently diagnosed incidentally at histologic assessment of the surgical specimen following appendectomy for suspected or diagnosed appendicitis(5). Despite being a rare disease, AA has various histological subtypes with distinct clinical behaviors such as mucinous adenocarcinoma, colonic subtype (non-mucinous adenocarcinoma) and signet-ring adenocarcinoma(6-9). Colonic subtype behaves like colon cancer clinically, whereas mucinous subtype often involves peritoneal cavity and rarely metastasizes outside of peritoneal cavity $(6,7)$. Signet-ring 
adenocarcinoma subtype is usually more aggressive and has the worst survival. Despite differences in stage distribution, cancer specific survivals are similar for mucinous and non-mucinous AA(8). Up to half of the AA patients present with advanced stage disease and in the advanced setting clinical management can be unique based on histological subtype with different survival outcomes between subtypes(6). In the absence of distant metastasis, right hemicolectomy is recommended, with at least 12 lymph nodes typically considered adequate for accurate staging(7). Almost half of the AA patients present with locoregional disease with no established post-surgical management, which is usually extrapolated from colon cancer management $(6,7)$.

Adjuvant chemotherapy (AC) is standard of care in stage III colon adenocarcinoma and provides a 22$32 \%$ overall survival (OS) benefit, and a $30 \%$ relative risk reduction in disease recurrence(10). AC with 5fluorouracil/leucovorin or capecitabine with oxaliplatin is included in post-surgical management of stage III AA if deemed medically fit(7). This approach is extrapolated from management of stage III colon adenocarcinoma(11). Not all patients with stage II colon cancer benefit from AC, and it is crucial to identify high-risk subgroups that might benefit from $\mathrm{AC}(11)$. Clinico-pathological high risk features have been defined as pathologic T4 (pT4) tumors, perforation, lymphovascular invasion (LVI), perineural invasion (PNI), less than 12 lymph nodes examined, high-grade tumors, positive margins, and obstruction(12-15). High-risk features are frequently utilized in AC decisions in stage Il colon cancer(13). Despite lack of definitive evidence from randomized trials, several mostly retrospective studies suggested that stage II colon cancer with high-risk features might achieve improved survival with AC(14-19). Similar to colon adenocarcinoma, AC is also often considered for stage II AA patients with high-risk features, especially younger patients and those with inadequate nodal staging(7). This approach is extrapolated from management of stage II colorectal cancer (CC) and the exact role of high-risk features is less established in stage II AA. The aim of this study is to determine the impact of high-risk features on clinical outcomes and whether high-risk features are predictive of AC benefit in stage II AA.

\section{Methods}

Patients with pathological stage II AA between 2010 and 2015 who underwent surgery were identified from the National Cancer Database (NCDB). The NCDB contains clinical and demographic information on $70 \%$ of all incident cancers in the United States from $>1500$ Commission-on-Cancer-accredited cancer centers. It is a joint quality improvement initiative of the American College of Surgeons Commission on Cancer and the American Cancer Society. Eligibility was obtained using the following ICD-0-3 morphology and topography codes were utilized: 8140/3 (adenocarcinoma), 8480/3 (mucinous adenocarcinoma) and C18.1 (Fig. 1). All other histology types such as goblet/ex-goblet cell, neuroendocrine tumor etc. were excluded. Patients treated with neoadjuvant systemic and/or radiation therapy and adjuvant radiation were excluded. High-risk was defined as having at least one of the following clinico-pathological features: T4 tumor, < 12 lymph nodes examined, poorly differentiated histology, positive margins, or

lymphovascular invasion. Patients with none of the above features were defined as low-risk. The primary outcome was overall survival (OS) of patients with high-risk features compared to low-risk features. The secondary outcome was OS of patients who received adjuvant chemotherapy compared to patients that 
received no treatment stratified by risk group. The following patient-specific covariates were included: sex, race, age at diagnosis, AJCC pathologic stage, microsatellite stability status, nodes examined, primary payor, chemotherapy, surgery of primary site, tumor size, and Charlson-Deyo score (Table 1).

Microsatellite stability status was divided into microsatellite stable (MSS) which included MSI stable (code 020) and MSI unstable low positive (code 040). Microsatellite instable (MSI-H) status included MSI unstable high positive (code 050) and MSI unstable positive (code 060). No ethical approval was required for the study as de-identified patient information in the NCDB is legally accessible to the public. 
Table 1

Baseline clinicopathological characteristics

\begin{tabular}{|c|c|c|}
\hline \multirow[t]{2}{*}{ Variable } & \multirow[t]{2}{*}{ Level } & Entire cohort \\
\hline & & $N(\%)=1040$ \\
\hline \multirow[t]{2}{*}{ Sex } & Male & $530(51.0)$ \\
\hline & Female & $510(49.0)$ \\
\hline \multirow[t]{3}{*}{ Race } & Caucasian & $879(84.5)$ \\
\hline & African American & $126(12.1)$ \\
\hline & Other/Unknown & $35(3.4)$ \\
\hline \multirow[t]{2}{*}{ Histology } & Adenocarcinoma (not otherwise specified) & $484(46.5)$ \\
\hline & Mucinous adenocarcinoma & $556(53.5)$ \\
\hline \multirow[t]{4}{*}{ Grade } & Well differentiated & $343(33.0)$ \\
\hline & Moderately differentiated & $565(54.3)$ \\
\hline & Poorly differentiated & $119(11.4)$ \\
\hline & Undifferentiated & $13(1.3)$ \\
\hline \multirow[t]{2}{*}{ Risk Group } & Low & $557(53.6)$ \\
\hline & High & $483(46.4)$ \\
\hline \multirow[t]{3}{*}{ Microsatellite Instability (MSI) } & MSI stable & $152(14.6)$ \\
\hline & MSI high & $14(1.3)$ \\
\hline & Undetermined & $874(84.0)$ \\
\hline \multirow[t]{5}{*}{ AJCC Pathologic T } & p2 & $4(0.4)$ \\
\hline & p3 & $547(52.6)$ \\
\hline & $\mathrm{p} 4$ & $62(6.0)$ \\
\hline & $\mathrm{p} 4 \mathrm{~A}$ & $280(26.9)$ \\
\hline & $\mathrm{p} 4 \mathrm{~B}$ & $147(14.1)$ \\
\hline \multirow[t]{3}{*}{ AJCC Pathologic N } & p0 & $967(93.0)$ \\
\hline & p1 & $1(0.1)$ \\
\hline & $\mathrm{pX}$ & $72(6.9)$ \\
\hline
\end{tabular}




\begin{tabular}{|c|c|c|}
\hline Variable & Level & $\begin{array}{l}\text { Entire cohort } \\
N(\%)=1040\end{array}$ \\
\hline \multirow[t]{4}{*}{ AJCC Pathologic M } & $\mathrm{p} 1$ & $6(0.6)$ \\
\hline & $\mathrm{p} 1 \mathrm{~A}$ & $5(0.5)$ \\
\hline & $\mathrm{p} 1 \mathrm{~B}$ & $1(0.1)$ \\
\hline & $\mathrm{pX}$ & $1028(98.8)$ \\
\hline \multirow[t]{2}{*}{ Lymph Nodes Examined } & $<12$ & $313(30.1)$ \\
\hline & $\geq 12$ & $727(69.9)$ \\
\hline \multirow[t]{2}{*}{ Lymphovascular Invasion } & Not present & $931(89.5)$ \\
\hline & Present & $109(10.5)$ \\
\hline \multirow[t]{2}{*}{ Year of Diagnosis } & $2010-2012$ & $439(42.2)$ \\
\hline & $2013-2015$ & $601(57.8)$ \\
\hline \multirow[t]{5}{*}{ Facility Type } & Community Cancer Program & $95(9.1)$ \\
\hline & Comprehensive Community Cancer Program & $435(41.8)$ \\
\hline & Academic/Research Program & $269(25.9)$ \\
\hline & Integrated Network Cancer Program & $165(15.9)$ \\
\hline & Unknown & $76(7.3)$ \\
\hline \multirow[t]{3}{*}{ Insurance Status } & Unknown/Not Insured & $63(6.1)$ \\
\hline & Private Insurance & $536(51.5)$ \\
\hline & Government Insurance & $441(42.4)$ \\
\hline \multirow[t]{3}{*}{ Charlson-Deyo Score } & 0 & $818(78.7)$ \\
\hline & 1 & $160(15.4)$ \\
\hline & $2+$ & $62(6.0)$ \\
\hline \multirow[t]{3}{*}{ Tumor Size $(\mathrm{cm})$} & $<5$ & $601(57.8)$ \\
\hline & $\geq 5$ & $273(26.3)$ \\
\hline & Unknown & $166(16.0)$ \\
\hline \multirow[t]{3}{*}{ Chemotherapy } & No & $637(61.3)$ \\
\hline & Yes & 369 (35.5) \\
\hline & Unknown & $34(3.3)$ \\
\hline
\end{tabular}




\begin{tabular}{|lll|}
\hline Variable & Level & Entire cohort \\
\hline Chemotherapy Treatment & No (\%) =1040 \\
& Number \& type unknown & $637(61.3)$ \\
& Single-agent chemotherapy & $20(1.9)$ \\
& Multiagent chemotherapy & $108(10.4)$ \\
& Unknown & $241(23.2)$ \\
\hline Surgery at Primary Site & Appendectomy & $34(3.3)$ \\
& Right hemicolectomy & $305(29.3)$ \\
& Total colectomy & $643(61.8)$ \\
& Surgery NOS & $33(3.2)$ \\
Surgical Margin & Negative & $59(5.7)$ \\
& Positive & $934(89.8)$ \\
& Not evaluable & $93(8.9)$ \\
& Mean & $13(1.3)$ \\
\hline Age at Diagnosis & Median & 60.48 \\
& Minimum & 61.00 \\
& Maximum & 19.00 \\
& Std Dev & 90.00 \\
& & 13.86 \\
\hline
\end{tabular}

\section{Statistical Analysis}

Eligible patients' demographic and disease information were described with appropriate descriptive statistics. Numeric variables were presented with mean, standard deviation, median and range, while categorical variables were presented with frequency and percentage. ANOVA tests were conducted for comparisons of numeric covariates, while Chi-square tests were utilized for categorical covariates. Univariate (UVA) and Multivariate (MVA) analyses and Cox Proportional-Hazards models were fitted to explore the association between each covariate and OS, which is defined as months from diagnosis to death or last contact. High risk group, chemotherapy, microsatellite status, sex, Charlson-Deyo score, race, year of diagnosis, tumor size, facility type, insurance status, and age at diagnosis were included in UVA and MVA models, with a backward elimination approach with the alpha level of 0.20 for covariate selections in MVA. Kaplan-Meier curves were also generated to compare UVA association with OS, with Log-rank test determining statistical significances. All analyses were done using SAS 9.4 (SAS Institute, Inc., Cary, North Carolina) with a significant level of 0.05 . 


\section{Results}

\section{Patient Demographics and Tumor Characteristics}

A total of 1,040 patients with resected pathological stage II AA were identified (Fig. 1). Baseline clinicopathological characteristics are summarized in Table 1. The median age at diagnosis was 61 (range, 19-90) years, with males accounting for $51 \%$. About $84.5 \%$ were Caucasian and distribution across high and low risk status was $46.4 \%(\mathrm{~N}=483)$ and $53.6 \%(\mathrm{~N}=557)$, respectively. MSS accounted for $14.6 \%$; MSI-H $1.3 \%$; MSI status undetermined $84 \% .50 .9 \%$ of the patients were treated at community practices and $25.9 \%$ were treated at academic or research cancer centers. Insurance coverage was mostly private $(51.5 \%)$ in comparison to government insurance (42.4\%) and no insurance (6.1\%). A higher number of patients were diagnosed between 2013-2015 (57.8\%) compared to 2010-2012 (42.2\%). Charlson-Deyo score was 0 for most patients (78.7\%) compared to $\geq 1$ in $21.4 \%$ of patients. Tumors were $<5 \mathrm{~cm}$ in $57.8 \%$ of cases and $\geq 5 \mathrm{~cm}$ in $26.3 \%$ (Table 1 ).

MSI-H accounted for $1.26 \%$ of low risk and $1.45 \%$ of high-risk tumors $(p=0.008)$. Covariates such as year of diagnosis, primary payor, sex, race, age at diagnosis and Charlson-Deyo score demonstrated similar proportions amongst high and low risk groups. Baseline clinicopathological characteristics stratified by risk status are summarized in Table 2. 
Table 2

Univariate Association with Risk Status

\begin{tabular}{|c|c|c|c|c|}
\hline Covariate & Level & $\begin{array}{l}\text { Low Risk } \\
\text { Group } \\
N(\%)= \\
557\end{array}$ & $\begin{array}{l}\text { High Risk } \\
\text { Group } \\
\mathrm{N}(\%)= \\
483\end{array}$ & $\begin{array}{l}\text { Parametric } \\
\text { P-Value }\end{array}$ \\
\hline Sex & $\begin{array}{l}\text { Male } \\
\text { Female }\end{array}$ & $\begin{array}{l}281 \\
(50.45) \\
276 \\
(49.55)\end{array}$ & $\begin{array}{l}249 \\
(51.55) \\
234 \\
(48.45)\end{array}$ & 0.481 \\
\hline Race & $\begin{array}{l}\text { Caucasian } \\
\text { African American } \\
\text { Other }\end{array}$ & $\begin{array}{l}476 \\
(85.46) \\
62(11.13) \\
19(3.41)\end{array}$ & $\begin{array}{l}403 \\
(83.44) \\
64(13.25) \\
16(3.31)\end{array}$ & 0.579 \\
\hline Year of Diagnosis & $\begin{array}{l}2010-2012 \\
2013-2015\end{array}$ & $\begin{array}{l}228 \\
(40.93) \\
329 \\
(59.07)\end{array}$ & $\begin{array}{l}211 \\
(43.69) \\
272 \\
(56.31)\end{array}$ & 0.370 \\
\hline Facility Type & $\begin{array}{l}\text { Community Cancer Program } \\
\text { Comprehensive Community } \\
\text { Cancer Program } \\
\text { Academic/Research Program } \\
\text { Integrated Network Cancer } \\
\text { Program } \\
\text { Unknown }\end{array}$ & $\begin{array}{l}44(7.90) \\
224 \\
(40.22) \\
149 \\
(26.75) \\
97(17.41) \\
43(7.72)\end{array}$ & $\begin{array}{l}51(10.56) \\
211 \\
(43.69) \\
120 \\
(24.84) \\
68(14.08) \\
33(6.83)\end{array}$ & 0.267 \\
\hline Insurance Status & $\begin{array}{l}\text { Not Insured/Unknown } \\
\text { Private Insurance } \\
\text { Government Insurance }\end{array}$ & $\begin{array}{l}28(5.03) \\
292 \\
(52.42) \\
237 \\
(42.55)\end{array}$ & $\begin{array}{l}35(7.25) \\
244 \\
(50.52) \\
204 \\
(42.24)\end{array}$ & 0.318 \\
\hline Charlson-Deyo Score & $\begin{array}{l}0 \\
1 \\
2+\end{array}$ & $\begin{array}{l}443 \\
(79.53) \\
81(14.54) \\
33(5.92)\end{array}$ & $\begin{array}{l}375 \\
(77.64) \\
79(16.36) \\
29(6.00)\end{array}$ & 0.714 \\
\hline
\end{tabular}




\begin{tabular}{|c|c|c|c|c|}
\hline \multirow[t]{2}{*}{ Covariate } & \multirow[t]{2}{*}{ Level } & $\begin{array}{l}\text { Low Risk } \\
\text { Group }\end{array}$ & $\begin{array}{l}\text { High Risk } \\
\text { Group }\end{array}$ & \multirow[t]{2}{*}{$\begin{array}{l}\text { Parametric } \\
\text { P-Value }\end{array}$} \\
\hline & & $\begin{array}{l}N(\%)= \\
557\end{array}$ & $\begin{array}{l}N(\%)= \\
483\end{array}$ & \\
\hline \multirow[t]{5}{*}{ Tumor Size (mm) } & Mean & 43.73 & 40.57 & \multirow[t]{5}{*}{0.336} \\
\hline & Median & 35 & 30 & \\
\hline & Minimum & 1 & 1 & \\
\hline & Maximum & 665 & 989 & \\
\hline & Std Dev & 41.84 & 55.13 & \\
\hline \multirow[t]{6}{*}{ Chemotherapy } & No & $337(60.5)$ & 300 & \multirow[t]{6}{*}{0.596} \\
\hline & Number \& type unknown & $9(1.62)$ & (0z.1) & \\
\hline & Single-agent chemotherapy & $65(11.67)$ & & \\
\hline & Multiagent chemotherapy & 129 & & \\
\hline & \multirow[t]{2}{*}{ Unknown } & $(\angle 0.1)$ & $(23.19)$ & \\
\hline & & ) & $17(3.52)$ & \\
\hline \multirow{3}{*}{$\begin{array}{l}\text { Microsatellite } \\
\text { Instability (MSI) }\end{array}$} & MSI stable & $99(17.77)$ & $53(10.97)$ & \multirow[t]{3}{*}{0.008} \\
\hline & MSI high & $7(1.26)$ & $7(1.45)$ & \\
\hline & Undetermined & $\begin{array}{l}451 \\
(80.97)\end{array}$ & $\begin{array}{l}423 \\
(87.58)\end{array}$ & \\
\hline \multirow[t]{5}{*}{ Age at Diagnosis } & Mean & 60.18 & 60.82 & \multirow[t]{5}{*}{0.456} \\
\hline & Median & 62 & 61 & \\
\hline & Minimum & 19 & 21 & \\
\hline & Maximum & 90 & 90 & \\
\hline & Std Dev & 13.43 & 14.35 & \\
\hline
\end{tabular}

\section{Risk Status and Overall Survival}

In the total cohort, high-risk status was associated with worse OS compared to low-risk in univariate (HR $1.55 ; 95 \% \mathrm{Cl} 1.18-2.02 ; \mathrm{p}=0.001)$ and multivariable analyses (HR 1.36; 95\% $\mathrm{Cl} 1.03-1.79 ; \mathrm{p}=0.028)$ (Table 3). High-risk stage II AA patients had significantly worse 5-year OS compared to low-risk patients (67.1\% vs. $74.5 \%, p=0.0013$ ) (Fig. 2). To adjust for the confounding effect of chemotherapy, a separate analysis was conducted to compare high and low-risk status among patients who only underwent surgery without being treated with chemotherapy. In patients who only underwent resection, high-risk status was still associated with worse OS compared to low-risk in univariate analysis (HR $1.62 ; 95 \% \mathrm{Cl}$ 1.15-2.29; $p=0.006)$ and a similar direction was seen on multivariable analysis, without reaching 
statistical significance (HR 1.32; 95\% Cl 0.91-1.90; $\mathrm{p}=0.147$ ) (Supplemental Table 1). High-risk patients still had significantly worse 5-year OS compared to low-risk patients after eliminating chemotherapy as a confounding variable $(66.5 \%$ vs. $76.3 \%, p=0.0057)$ (Fig. 3$)$. 
Table 3

Univariate and Multivariate Association with Overall Survival (Total Cohort)

\begin{tabular}{|c|c|c|c|}
\hline Covariate & Level & $\begin{array}{l}\text { Univariate Analysis } \\
\text { Hazard Ratio (95\% } \\
\text { Cl) p-value }\end{array}$ & $\begin{array}{l}\text { Multivariate } \\
\text { Analysis } \\
\text { Hazard Ratio ( } 95 \% \\
\text { Cl) p-value }\end{array}$ \\
\hline Risk Status & $\begin{array}{l}\text { High Risk } \\
\text { Low Risk* }\end{array}$ & $\begin{array}{l}1.55 ; 1.18-2.02 ; p= \\
0.001\end{array}$ & $\begin{array}{l}1.36 ; 1.03-1.79 ; p= \\
0.028\end{array}$ \\
\hline Chemotherapy & $\begin{array}{l}\text { Yes } \\
\text { Unknown } \\
\text { No* }\end{array}$ & $\begin{array}{l}0.88 ; 0.67-1.17 ; p= \\
0.380 \\
1.18 ; 0.58-2.41 ; p= \\
0.648\end{array}$ & - \\
\hline $\begin{array}{l}\text { Microsatellite } \\
\text { Instability (MSI) }\end{array}$ & $\begin{array}{l}\text { MSI high } \\
\text { Undetermined } \\
\text { MSI stable* }\end{array}$ & $\begin{array}{l}0.82 ; 0.20-3.44 ; p= \\
0.786 \\
1.03 ; 0.70-1.53 ; p= \\
0.873\end{array}$ & - \\
\hline Sex & $\begin{array}{l}\text { Female } \\
\text { Male* }\end{array}$ & $\begin{array}{l}0.80 ; 0.61-1.04 ; p= \\
0.096\end{array}$ & $\begin{array}{l}0.75 ; 0.57-0.98 ; p= \\
0.037\end{array}$ \\
\hline Race & $\begin{array}{l}\text { African American } \\
\text { Other } \\
\text { Caucasian* }\end{array}$ & $\begin{array}{l}1.37 ; 0.92-2.04 ; p= \\
0.124 \\
1.26 ; 0.62-2.56 ; p= \\
0.521\end{array}$ & $\begin{array}{l}1.69 ; 1.12-2.55 ; p= \\
0.013 \\
1.18 ; 0.58-2.42 ; p= \\
0.647\end{array}$ \\
\hline Year of Diagnosis & $\begin{array}{l}2010-2012 \\
2013-2015^{\star}\end{array}$ & $\begin{array}{l}0.96 ; 0.71-1.29 ; p= \\
0.782\end{array}$ & - \\
\hline Facility Type & $\begin{array}{l}\text { Community Cancer Program } \\
\text { Academic/Research Program } \\
\text { Integrated Network Cancer } \\
\text { Program } \\
\text { Unknown } \\
\text { Comprehensive Community } \\
\text { Cancer Program }\end{array}$ & $\begin{array}{l}0.91 ; 0.56-1.47 ; p= \\
0.690 \\
0.89 ; 0.64-1.24 ; p= \\
0.495 \\
0.97 ; 0.66-1.43 ; p= \\
0.894 \\
0.43 ; 0.21-0.89 ; p= \\
0.023\end{array}$ & - \\
\hline Insurance Status & $\begin{array}{l}\text { Not Insured/Unknown } \\
\text { Government Insurance } \\
\text { Private* }\end{array}$ & $\begin{array}{l}0.96 ; 0.50-1.85 ; p= \\
0.902 \\
2.23 ; 1.69-2.96 ; p< \\
0.001\end{array}$ & - \\
\hline
\end{tabular}




\begin{tabular}{|c|c|c|c|}
\hline \multirow[t]{2}{*}{ Covariate } & \multirow[t]{2}{*}{ Level } & Univariate Analysis & \multirow{2}{*}{$\begin{array}{l}\text { Multivariate } \\
\text { Analysis } \\
\text { Hazard Ratio (95\% } \\
\text { Cl) p-value }\end{array}$} \\
\hline & & $\begin{array}{l}\text { Hazard Ratio ( } 95 \% \\
\text { Cl) p-value }\end{array}$ & \\
\hline \multirow{3}{*}{$\begin{array}{l}\text { Charlson-Deyo } \\
\text { Score }\end{array}$} & 1 & $1.32 ; 0.93-1.88 ; p=$ & \multirow[t]{3}{*}{-} \\
\hline & $2+$ & & \\
\hline & $0^{*}$ & $\begin{array}{l}2.23 ; 1.45-3.42 ; p< \\
0.001\end{array}$ & \\
\hline \multirow[t]{3}{*}{ Tumor Size (cm) } & & $\begin{array}{l}0.88 ; 0.64-1.21 ; p= \\
0.419\end{array}$ & \multirow[t]{3}{*}{-} \\
\hline & Unknown & $1.95: 0.80-4.74: p=$ & \\
\hline & $<5^{\star}$ & 0.142 & \\
\hline \multirow[t]{2}{*}{ Age at Diagnosis } & $>65$ & \multirow{2}{*}{$\begin{array}{l}3.01 ; 2.30-3.95 ; p< \\
0.001\end{array}$} & \multirow[t]{2}{*}{-} \\
\hline & $\leq 65^{\star}$ & & \\
\hline *Reference & & & \\
\hline
\end{tabular}

\section{Adjuvant Chemotherapy in High and Low Risk Stage II AA}

AC was administered in $34.4 \%(n=166)$ of high-risk patients and in $36.5 \%(n=203)$ of low-risk patients $(p=0.742)$ (Table 2). Among high-risk patients, AC was not associated with better OS in univariate (HR $0.86 ; 95 \% \mathrm{Cl} 0.59-1.26 ; \mathrm{p}=0.448$ and multivariable analyses $(\mathrm{HR} 1.35 ; 95 \% \mathrm{Cl} 0.90-2.04 ; \mathrm{p}=0.151$ compared to no AC (Table 4). Similarly, among low-risk patients, AC was not associated with better OS in univariate (HR 0.92; 95\% $\mathrm{Cl} 0.60-1.39 ; \mathrm{p}=0.679$ and multivariable analyses (HR 1.27; 95\% $\mathrm{Cl} 0.81-2.02$; $p=0.299$ ) compared to no AC (Table 5). For high-risk patients, 5 -year OS was $68.3 \%$ in patients that received AC vs. $66.5 \%$ in patients that did not $(p=0.722)$ (Fig. 4). For low-risk patients, 5 -year OS was $74.0 \%$ in patients that received AC vs. $76.3 \%$ in patients that did not $(p=0.813)$ (Fig. 5). 
Table 4

Univariate and Multivariate Association with Overall Survival (High Risk)

\begin{tabular}{|c|c|c|c|}
\hline Covariate & Level & $\begin{array}{l}\text { High Risk } \\
\text { Univariate Analysis } \\
\text { Hazard Ratio ( } 95 \% \\
\text { Cl) p-value }\end{array}$ & $\begin{array}{l}\text { High Risk } \\
\text { Multivariate } \\
\text { Analysis } \\
\text { Hazard Ratio (95\% } \\
\text { Cl) p-value }\end{array}$ \\
\hline Chemotherapy & & $\begin{array}{l}0.86 ; 0.59-1.26 ; p \\
=0.448\end{array}$ & $\begin{array}{l}1.35 ; 0.90-2.04 ; p= \\
0.151\end{array}$ \\
\hline & No* & $\begin{array}{l}1.09 ; 0.40-2.99 ; p \\
=0.865\end{array}$ & $\begin{array}{l}1.41 ; 0.51-3.89 ; p= \\
0.512\end{array}$ \\
\hline $\begin{array}{l}\text { Microsatellite } \\
\text { Instability (MSI) }\end{array}$ & $\begin{array}{l}\text { MSI high } \\
\text { Undetermined } \\
\text { MSI stable* }\end{array}$ & $\begin{array}{l}1.21 ; 0.27-5.31 ; \mathrm{p} \\
=0.803 \\
0.83 ; 0.48-1.46 ; \mathrm{p} \\
=0.522\end{array}$ & - \\
\hline Sex & $\begin{array}{l}\text { Female } \\
\text { Male* }\end{array}$ & $\begin{array}{l}0.67 ; 0.47-0.97 ; p \\
=0.032\end{array}$ & $\begin{array}{l}0.63 ; 0.44-0.91 ; p= \\
0.014\end{array}$ \\
\hline Race & $\begin{array}{l}\text { African American } \\
\text { Other } \\
\text { Caucasian* }\end{array}$ & $\begin{array}{l}0.41 ; 0.85-2.33 ; \mathrm{p} \\
=0.181 \\
1.47 ; 0.60-3.61 ; \mathrm{p} \\
=0.402\end{array}$ & $\begin{array}{l}2.01 ; 1.19-3.38 ; p= \\
0.009 \\
1.28 ; 0.51-3.21 ; p= \\
0.593\end{array}$ \\
\hline Year of Diagnosis & $\begin{array}{l}2010-2012 \\
2013-2015^{\star}\end{array}$ & $\begin{array}{l}0.83 ; 0.57-1.21 ; p \\
=0.332\end{array}$ & - \\
\hline Facility Type & $\begin{array}{l}\text { Community Cancer Program } \\
\text { Academic/Research Program } \\
\text { Integrated Network Cancer } \\
\text { Program } \\
\text { Unknown } \\
\text { Comprehensive Community } \\
\text { Cancer Program* }\end{array}$ & $\begin{array}{l}0.84 ; 0.45-1.57 ; p \\
=0.595 \\
1.09 ; 0.71-1.69 ; p \\
=0.682 \\
0.89 ; 0.52-1.53 ; p \\
=0.683 \\
0.31 ; 0.10-1.00 ; p= \\
0.050\end{array}$ & - \\
\hline Insurance Status & $\begin{array}{l}\text { Not Insured/Unknown } \\
\text { Government Insurance } \\
\text { Private* }\end{array}$ & $\begin{array}{l}1.03 ; 0.46-2.28 ; \mathrm{p} \\
=0.948 \\
2.28 ; 1.56-3.33 ; \mathrm{p} \\
<0.001\end{array}$ & - \\
\hline
\end{tabular}




\begin{tabular}{|c|c|c|c|}
\hline Covariate & Level & $\begin{array}{l}\text { High Risk } \\
\text { Univariate Analysis } \\
\text { Hazard Ratio (95\% } \\
\text { Cl) p-value }\end{array}$ & $\begin{array}{l}\text { High Risk } \\
\text { Multivariate } \\
\text { Analysis } \\
\text { Hazard Ratio (95\% } \\
\text { Cl) p-value }\end{array}$ \\
\hline $\begin{array}{l}\text { Charlson-Deyo } \\
\text { Score }\end{array}$ & $\begin{array}{l}1 \\
2+ \\
0 *\end{array}$ & $\begin{array}{l}1.52 ; 0.98-2.35 ; p \\
=0.059 \\
2.11 ; 1.15-3.88 ; p \\
=0.015\end{array}$ & - \\
\hline Tumor Size (cm) & $\begin{array}{l}\geq 5 \\
\text { Unknown } \\
<5^{\star}\end{array}$ & $\begin{array}{l}0.87 ; 0.56-1.36 ; p \\
=0.538 \\
0.60 ; 0.36-0.99 ; p \\
=0.048\end{array}$ & - \\
\hline Age at Diagnosis & $\begin{array}{l}>65 \\
\leq 65^{*}\end{array}$ & $\begin{array}{l}3.21 ; 2.23-4.61 ; p \\
<0.001\end{array}$ & - \\
\hline *Reference & & & \\
\hline
\end{tabular}


Table 5

Univariate and Multivariate Association with Overall Survival (Low Risk)

\begin{tabular}{|c|c|c|c|}
\hline Covariate & Level & $\begin{array}{l}\text { Low Risk Univariate } \\
\text { Analysis } \\
\text { Hazard Ratio (95\% } \\
\text { Cl) p-value }\end{array}$ & $\begin{array}{l}\text { Low Risk } \\
\text { Multivariate } \\
\text { Analysis } \\
\text { Hazard Ratio (95\% } \\
\text { Cl) p-value }\end{array}$ \\
\hline Chemotherapy & & $\begin{array}{l}0.92 ; 0.60-1.39 ; p \\
=0.679\end{array}$ & $\begin{array}{l}1.27 ; 0.81-2.02 ; p= \\
0.299\end{array}$ \\
\hline & No* & $\begin{array}{l}1.24 ; 0.45-3.43 ; p \\
=0.676\end{array}$ & $\begin{array}{l}1.97 ; 0.68-5.70 ; p= \\
0.210\end{array}$ \\
\hline $\begin{array}{l}\text { Microsatellite } \\
\text { Instability (MSI) }\end{array}$ & $\begin{array}{l}\text { MSI high } \\
\text { Undetermined } \\
\text { MSI stable* }\end{array}$ & $\begin{array}{l}0.00 ; 0.00-. ; p= \\
0.984 \\
1.12 ; 0.64-1.94 ; p \\
=0.697\end{array}$ & - \\
\hline Sex & $\begin{array}{l}\text { Female } \\
\text { Male* }^{\star}\end{array}$ & $\begin{array}{l}0.99 ; 0.67-1.49 ; p \\
=0.980\end{array}$ & - \\
\hline Race & $\begin{array}{l}\text { African American } \\
\text { Other } \\
\text { Caucasian* }\end{array}$ & $\begin{array}{l}1.26 ; 0.65-2.44 ; \mathrm{p} \\
=0.493 \\
1.12 ; 0.35-3.54 ; \\
0.852\end{array}$ & - \\
\hline Year of Diagnosis & $\begin{array}{l}2010-2012 \\
2013-2015^{\star}\end{array}$ & $\begin{array}{l}1.12 ; 0.70-1.79 ; p \\
=0.628\end{array}$ & - \\
\hline Facility Type & $\begin{array}{l}\text { Community Cancer Program } \\
\text { Academic/Research Program } \\
\text { Integrated Network Cancer } \\
\text { Program }\end{array}$ & $\begin{array}{l}0.98 ; 0.46-2.09 ; \mathrm{p} \\
=0.963 \\
0.72 ; 0.43-1.21 ; \mathrm{p} \\
=0.214 \\
1.15 ; 0.66-1.99 ; \mathrm{p}\end{array}$ & $\begin{array}{l}1.27 ; 0.58-2.77 ; p= \\
0.551 \\
0.60 ; 0.35-1.04 ; p= \\
0.070 \\
0.88 ; 0.50-1.54 ; p=\end{array}$ \\
\hline & $\begin{array}{l}\text { Comprehensive Community } \\
\text { Cancer Program }\end{array}$ & $\begin{array}{l}0.59 ; 0.23-1.50 ; p \\
=0.270\end{array}$ & $\begin{array}{l}2.66 ; 0.82-8.63 ; p= \\
0.104\end{array}$ \\
\hline Insurance Status & $\begin{array}{l}\text { Not Insured/Unknown } \\
\text { Government Insurance } \\
\text { Private* }\end{array}$ & $\begin{array}{l}0.78 ; 0.24-2.52 ; p \\
=0.676 \\
2.20 ; 1.45-3.33 ; p \\
<0.001\end{array}$ & - \\
\hline
\end{tabular}




\begin{tabular}{|c|c|c|c|}
\hline Covariate & Level & $\begin{array}{l}\text { Low Risk Univariate } \\
\text { Analysis } \\
\text { Hazard Ratio (95\% } \\
\text { Cl) p-value }\end{array}$ & $\begin{array}{l}\text { Low Risk } \\
\text { Multivariate } \\
\text { Analysis } \\
\text { Hazard Ratio (95\% } \\
\text { Cl) p-value }\end{array}$ \\
\hline \multirow[t]{2}{*}{$\begin{array}{l}\text { Charlson-Deyo } \\
\text { Score }\end{array}$} & 1 & $\begin{array}{l}1.04 ; 0.56-1.91 ; p \\
=0.908\end{array}$ & $\begin{array}{l}0.57 ; 0.29-1.12 ; p= \\
0.102\end{array}$ \\
\hline & $0 *$ & $\begin{array}{l}2.35 ; 1.27-4.33 ; p \\
=0.006\end{array}$ & $\begin{array}{l}1.76 ; 0.92-3.35 ; p= \\
0.088\end{array}$ \\
\hline \multirow[t]{2}{*}{ Tumor Size (cm) } & $\geq 5$ & \multirow{2}{*}{$\begin{array}{l}0.96 ; 0.61-1.51 ; p \\
=0.857 \\
0.80 ; 0.42-1.52 ; p \\
=0.487\end{array}$} & \multirow[t]{2}{*}{-} \\
\hline & $\begin{array}{l}\text { Unknown } \\
<5^{\star}\end{array}$ & & \\
\hline Age at Diagnosis & $\begin{array}{l}>65 \\
\leq 65^{*}\end{array}$ & $\begin{array}{l}2.80 ; 1.86-4.20 ; p \\
<0.001\end{array}$ & - \\
\hline *Reference & & & \\
\hline
\end{tabular}

\section{Discussion}

Appendiceal adenocarcinoma is a rare malignancy with complex clinical management. Significant increase has been reported in annual incidence of all appendiceal tumors from 1973 to 2014(6). Almost half of the patients present with locoregional disease and post-surgical management is less established. Clinicopathological high-risk features of colon adenocarcinoma as described in national guidelines(13, $20,21)$ are often utilized in stage II appendiceal adenocarcinoma management despite lack of data(7). The presence of traditional "high-risk" pathologic features in stage II colon cancer can identify a subgroup of patients with a recurrence risk approximating that of stage III disease(22). The results in this study similarly demonstrated that patients with high-risk AA had significantly worse OS compared to their low-risk counterparts in multivariable analysis. High-risk stage II AA patients also had significantly worse 5-year OS compared to low-risk patients $(67.1 \%$ vs. $74.5 \%, p=0.0013)$. Thus, high-risk features are prognostic in stage II AA. In addition to clinico-pathological high-risk features high-quality prognostic biomarkers could also be useful clinically to estimate proper therapeutic strategies or to predict prognosis(23). A nomogram predict model proposed by Xie et al, who collected data for 1404 patients with adenocarcinoma of the appendix from the Surveillance, Epidemiology, and End Results Program (SEER) database, noted that different stages meant disparate therapies and discrepant prognosis between low- and high-risk patients, especially in stage I-III adenocarcinoma of the appendix(23). Their novel nomogram prognosis prediction model contained seven clinical factors (age, marital status, depth of tumor invasion, total number of resected regional lymph nodes, number of metastatic lymph nodes, histological grade and distant metastases), in patients who underwent surgical treatment, that were more accurate than those generated using the traditional AJCC TNM Staging system(23). However, due to the 
limitations of the SEER database, information regarding the administration of chemotherapy could not be obtained(23).

The American Society of Colon and Rectal Surgeons (ASCRS) clinical practice guidelines recommend that patients with non-metastatic adenocarcinoma of the appendix should undergo right hemicolectomy(24). However, there are no published guidelines regarding AC for stage II AA due to lack of prospective trials. Management of stage II AA is extrapolated from stage II CC but studies examining role of high-risk features in stage II AA are lacking in adjuvant setting. To the best of our knowledge, this study is the first to describe the prognostic use of clinicopathological high-risk features in stage II AA. The results of this study demonstrated that clinicopathological high-risk features are prognostic in stage II AA. Neither resected high or low-risk stage II AA benefit from AC compared to no treatment in this study. Asare et al. reported that patients with mucinous and non-mucinous stage II and III appendiceal AA benefited from adjuvant systemic chemotherapy in their study examining impact of mucinous histology, grade and stage on all stages of $A A(25)$. However, clinicopathological features of stage II AA patients who would benefit from adjuvant treatment was not studied.

Despite the novel findings of this study, this is a retrospective study with its inherent limitations. The limitations of this study include lack of specific chemotherapy regimen data, duration of chemotherapy and data about adverse effects of chemotherapy. The analysis was primarily based on receipt of any chemotherapy and does not account for early discontinuation of prescribed treatment, which possibly could impact the survival benefit. Certain high-risk features, such as clinical presentation with obstruction or perforation are not included in the NCDB. In addition, disease-specific mortality, recurrence indices, and response to treatment are not captured in the NCDB. Despite these limitations, this study is the largest collective series currently available for pathological stage II AA classified by low- and high-risk features. Furthermore, this study can assist clinicians with adjuvant treatment decisions in stage II AA.

\section{Conclusion}

High-risk stage II AA patients had significantly worse 5-year OS compared to low-risk patients. AC did not appear to improve survival regardless of high-risk features in stage II AA

\section{List Of Abbreviations}

Adjuvant chemotherapy (AC)

Appendiceal adenocarcinoma (AA)

National Cancer Database (NCDB)

Mixed neuroendocrine-non-neuroendocrine neoplasm (MiNEN)

Overall survival (OS) 
Pathologic T4 (pT4) tumors

Lymphovascular invasion (LVI)

Perineural invasion (PNI)

Colorectal cancer (CC)

Microsatellite stable (MSS)

Microsatellite instable (MSI-H)

Univariate analysis (UVA)

Multivariate analysis (MVA)

American Society of Colon and Rectal Surgeons (ASCRS)

\section{Declarations}

Ethics approval and consent to participate: No ethical approval was required for the study as de-identified patient information in the NCDB is legally accessible to the public.

Consent for publication: Not applicable.

Availability of data and materials: Part of data presented in this study was presented at the $2020 \mathrm{GI}$ ASCO Annual Meeting in San Francisco, CA. The manuscript's abstract was published in "Abstracts" in Journal of Clinical Oncology: J Clin Oncol 38, 2020 (suppl 4; abstr 795). Research reported in this publication was supported in part by the Winship Research Informatics Shared Resource of Winship Cancer Institute of Emory University and $\mathrm{NIH} / \mathrm{National}$ Cancer Institute under award numbers P30CA138292. The content is solely the responsibility of the authors and does not necessarily represent the official views of the NIH. The data used in the study are derived from a de-identified NCDB file. The NCDB is a joint project of the Commission on Cancer of the American College of Surgeons and the American Cancer Society. The American College of Surgeons and the Commission on Cancer have not verified and are not responsible for the analytic or statistical methodology employed, or the conclusions drawn from these data by the investigator.

Competing interests: The authors declare that they have no competing interests.

Funding: There was no specific funding for this study and there are no competing financial disclosures.

Authors' contributions: MA was involved in conceptualization, data curation, formal analysis, investigation, project administration, supervision, validation and writing - review and editing. KZ was involved in conceptualization, data curation, formal analysis, investigation, project administration, supervision, validation and writing - review and editing. MP was involved in data curation, methodology, 
software, writing - original draft, and writing - review and editing. RJ was involved in data curation, methodology, software, writing - original draft, and writing - review and editing. OBA was involved in the methodology, investigation, original draft, and writing - review and editing. WLS was involved in the methodology, investigation, original draft, and writing - review and editing. CW was involved in the methodology, investigation, original draft, and writing - review and editing. MB was involved in the conceptualization, project administration, investigation, validation, review and editing. BFER was involved in the methodology, investigation, original draft, and writing - review and editing. All authors read and approved the final manuscript.

Acknowledgements: Not applicable.

\section{References}

1. Shaib WL, Assi R, Shamseddine A, Alese OB, Staley C, 3rd, Memis B, et al. Appendiceal Mucinous Neoplasms: Diagnosis and Management. Oncologist. 2017;22(9):1107-16.

2. Nagtegaal ID, Odze RD, Klimstra D, Paradis V, Rugge M, Schirmacher $P$, et al. The 2019 WHO classification of tumours of the digestive system. Histopathology. 2020;76(2):182-8.

3. McCusker ME, Cote TR, Clegg LX, Sobin LH. Primary malignant neoplasms of the appendix: a population-based study from the surveillance, epidemiology and end-results program, 1973-1998. Cancer. 2002;94(12):3307-12.

4. Nitecki SS, Wolff BG, Schlinkert R, Sarr MG. The natural history of surgically treated primary adenocarcinoma of the appendix. Annals of surgery. 1994;219(1):51-7.

5. León Arellano M, González-Domínguez Y, Molina-Ortiz F, Garceau MAH, Cantero R, Rodríguez-Montes JA. Primary adenocarcinoma of the appendix: Experience at La Paz University Hospital of Madrid (1967-2014). International Journal of Surgery Open. 2016;4:23-6.

6. Mo S, Zhou Z, Ying Z, Dai W, Xiang W, Han L, et al. Epidemiology of and prognostic factors for appendiceal carcinomas: a retrospective, population-based study. Int J Colorectal Dis. 2019;34(11):1915-24.

7. Kelly KJ. Management of Appendix Cancer. Clin Colon Rectal Surg. 2015;28(4):247-55.

8. Overman MJ, Fournier K, Hu CY, Eng C, Taggart M, Royal R, et al. Improving the AJCC/TNM staging for adenocarcinomas of the appendix: the prognostic impact of histological grade. Annals of surgery. 2013;257(6):1072-8.

9. Assarzadegan N, Montgomery E. What is New in 2019 World Health Organization (WHO) Classification of Tumors of the Digestive System: Review of Selected Updates on Neuroendocrine Neoplasms, Appendiceal Tumors, and Molecular Testing. Archives of pathology \& laboratory medicine. 2020.

10. Benson AB, 3rd. Adjuvant chemotherapy of stage III colon cancer. Seminars in oncology. 2005;32(6 Suppl 9):S74-7. 
11. Kannarkatt J, Joseph J, Kurniali PC, Al-Janadi A, Hrinczenko B. Adjuvant Chemotherapy for Stage II Colon Cancer: A Clinical Dilemma. Journal of oncology practice. 2017;13(4):233-41.

12. Artac M, Turhal NS, Kocer M, Karabulut B, Bozcuk H, Yalcin S, et al. Do high-risk features support the use of adjuvant chemotherapy in stage II colon cancer? A Turkish Oncology Group study. Tumori. 2014;100(2):143-8.

13. Benson AB, 3rd, Schrag D, Somerfield MR, Cohen AM, Figueredo AT, Flynn PJ, et al. American Society of Clinical Oncology recommendations on adjuvant chemotherapy for stage II colon cancer. J Clin Oncol. 2004;22(16):3408-19.

14. Kumar A, Kennecke HF, Renouf DJ, Lim HJ, Gill S, Woods R, et al. Adjuvant chemotherapy use and outcomes of patients with high-risk versus low-risk stage II colon cancer. Cancer. 2015;121(4):52734.

15. Park JS, Chon HJ, Jeung HC, Shin SJ, Rha SY, Ahn JB, et al. High-risk clinicopathological features and their predictive significance in Korean patients with stage II colon cancer. Journal of cancer research and clinical oncology. 2016;142(9):2051-9.

16. Meyers BM, Cosby R, Quereshy F, Jonker D. Adjuvant systemic chemotherapy for stages II and III colon cancer after complete resection: a clinical practice guideline. Current oncology (Toronto, Ont). 2016;23(6):418-24.

17. Casadaban L, Rauscher G, Aklilu M, Villenes D, Freels S, Maker AV. Adjuvant chemotherapy is associated with improved survival in patients with stage II colon cancer. Cancer. 2016;122(21):327787.

18. Sato H, Maeda K, Sugihara K, Mochizuki H, Kotake K, Teramoto T, et al. High-risk stage II colon cancer after curative resection. Journal of surgical oncology. 2011;104(1):45-52.

19. Verhoeff SR, van Erning FN, Lemmens VE, de Wilt JH, Pruijt JF. Adjuvant chemotherapy is not associated with improved survival for all high-risk factors in stage II colon cancer. International journal of cancer. 2016;139(1):187-93.

20. Labianca R, Nordlinger B, Beretta GD, Mosconi S, Mandala M, Cervantes A, et al. Early colon cancer: ESMO Clinical Practice Guidelines for diagnosis, treatment and follow-up. Ann Oncol. 2013;24 Suppl 6:vi64-72.

21. National Comprehensive Cancer Network. Colon Cancer (Version 2.2020) [Available from: https://www.nccn.org/professionals/physician_gls/pdf/colon_blocks.pdf.

22. Dotan E, Cohen SJ. Challenges in the management of stage II colon cancer. Seminars in oncology. 2011;38(4):511-20.

23. Xie X, Zhou Z, Song Y, Li W, Diao D, Dang C, et al. The Management and Prognostic Prediction of Adenocarcinoma of Appendix. Sci Rep. 2016;6:39027.

24. Glasgow SC, Gaertner W, Stewart D, Davids J, Alavi K, Paquette IM, et al. The American Society of Colon and Rectal Surgeons, Clinical Practice Guidelines for the Management of Appendiceal Neoplasms. Diseases of the colon and rectum. 2019;62(12):1425-38. 
25. Asare EA, Compton CC, Hanna NN, Kosinski LA, Washington MK, Kakar S, et al. The impact of stage, grade, and mucinous histology on the efficacy of systemic chemotherapy in adenocarcinomas of the appendix: Analysis of the National Cancer Data Base. Cancer. 2016;122(2):213-21.

\section{Supplemental Table}




\begin{tabular}{|c|c|c|c|c|c|}
\hline \multirow{2}{*}{$\begin{array}{l}\text { Covariate } \\
\text { Risk Status }\end{array}$} & Level & \multicolumn{2}{|c|}{$\begin{array}{l}\text { Univariate Analysis } \\
\text { Hazard Ratio ( } 95 \% \text { CI) } \\
\text { p-value }\end{array}$} & \multicolumn{2}{|c|}{$\begin{array}{l}\text { Multivariate Analysis } \\
\text { Hazard Ratio (95\% CI) } \\
\text { p-value }\end{array}$} \\
\hline & $\begin{array}{l}\text { High Risk } \\
\text { Low Risk* }\end{array}$ & $\begin{array}{l}1.62 \\
\mathrm{p}=0.006\end{array}$ & 1.15-2.29; & $\begin{array}{l}1.32 \\
p=0.147\end{array}$ & 0.91-1.90; \\
\hline \multirow[t]{2}{*}{$\begin{array}{l}\text { Microsatellite } \\
\text { Instability (MSI) }\end{array}$} & $\begin{array}{l}\text { MSI high } \\
\text { Undetermined }\end{array}$ & $\begin{array}{l}0.73 \\
\mathrm{p}=0.760\end{array}$ & $.010-5.47$ & - & \\
\hline & MSI stable* & $\begin{array}{l}1.00 \\
p=0.996\end{array}$ & 0.61-1.65; & & \\
\hline Nodes Examined & $\begin{array}{l}<12 \\
\square 12^{*}\end{array}$ & $\begin{array}{l}1.62 \\
\mathrm{p}=0.006\end{array}$ & 1.15-2.28; & - & \\
\hline Surgical Margins & $\begin{array}{l}\text { Positive } \\
\text { Negative* }\end{array}$ & $\begin{array}{l}2.41 \\
\mathrm{p}<0.001\end{array}$ & 1.51-3.85; & - & \\
\hline Sex & $\begin{array}{l}\text { Female } \\
\text { Male* }\end{array}$ & $\begin{array}{l}0.70 \\
\mathrm{p}=0.045\end{array}$ & 0.50-0.99; & $\begin{array}{l}0.68 \\
p=0.041\end{array}$ & 0.48-0.98; \\
\hline \multirow[t]{2}{*}{ Race } & $\begin{array}{l}\text { African American } \\
\text { Other }\end{array}$ & $\begin{array}{l}1.39 \\
\mathrm{p}=0.192\end{array}$ & $0.85-2.26$ & $\begin{array}{l}2.06 \\
p=0.006\end{array}$ & 1.23-3.46; \\
\hline & Caucasian* & $\begin{array}{l}1.55 \\
p=0.296\end{array}$ & 0.68-3.54; & $\begin{array}{l}1.28 \\
p=0.566\end{array}$ & 0.55-3.00; \\
\hline Year of Diagnosis & $\begin{array}{l}2010-2012 \\
2013-2015^{*}\end{array}$ & $\begin{array}{l}1.17 \\
\mathrm{p}=0.407\end{array}$ & 0.80-1.71; & - & \\
\hline \multirow[t]{4}{*}{ Facility Type } & $\begin{array}{l}\text { Community Cancer Program } \\
\text { Academic/Research Program }\end{array}$ & $\begin{array}{l}0.86 \\
p=0.603\end{array}$ & 0.48-1.53; & $\begin{array}{l}1.05 \\
p=0.878\end{array}$ & 0.57-1.91; \\
\hline & $\begin{array}{l}\text { Integrated Network } \quad \text { Cancer } \\
\text { Program }\end{array}$ & $\begin{array}{l}0.83 \\
\mathrm{p}=0.413\end{array}$ & 0.54-1.29; & $\begin{array}{l}1.09 \\
p=0.711\end{array}$ & 0.69-1.74; \\
\hline & $\begin{array}{l}\text { Unknown } \\
\text { Comprehensive } \quad \text { Community }\end{array}$ & $\begin{array}{l}0.61 \\
\mathrm{p}=0.067\end{array}$ & 0.36-1.04; & $\begin{array}{l}0.56 \\
p=0.036\end{array}$ & 0.32-0.96; \\
\hline & Cancer Program* & $\begin{array}{l}0.38 \\
\mathrm{p}=0.102\end{array}$ & $0.12-1.21$ & $\begin{array}{l}4.27 \\
p=0.034\end{array}$ & 1.12-16.32; \\
\hline \multirow[t]{2}{*}{ Insurance Status } & $\begin{array}{l}\text { Not Insured/Unknown } \\
\text { Government Insurance }\end{array}$ & $\begin{array}{l}0.91 \\
\mathrm{p}=0.855\end{array}$ & 0.32-2.56; & - & \\
\hline & Private* & $\begin{array}{l}3.22 \\
\mathrm{p}<0.001\end{array}$ & 2.17-4.78; & & \\
\hline \multirow[t]{2}{*}{ Charlson-Deyo Score } & $\begin{array}{l}1 \\
2+\end{array}$ & $\begin{array}{l}1.26 \\
\mathrm{p}=0.320\end{array}$ & 0.80-1.98; & $\begin{array}{l}1.05 \\
\mathrm{p}=0.829\end{array}$ & 0.66-1.69; \\
\hline & $0^{*}$ & $\begin{array}{l}2.63 \\
\mathrm{p}<0.001\end{array}$ & 1.63-4.24; & $\begin{array}{l}1.84 \\
\mathrm{p}=0.017\end{array}$ & 1.11-3.03; \\
\hline \multirow[t]{2}{*}{ Tumor Size $(\mathrm{cm})$} & $\begin{array}{l}\geq 5 \\
\text { Unknown }\end{array}$ & $\begin{array}{l}1.05 \\
\mathrm{p}=0.822\end{array}$ & 0.70-1.57; & - & \\
\hline & $<5^{*}$ & $\begin{array}{l}0.55 \\
\mathrm{p}=0.045\end{array}$ & 0.31-0.99; & & \\
\hline Age at Diagnosis & $>65$ & 3.90 & 2.67-5.68; & - & \\
\hline
\end{tabular}


*Reference

Supplemental Table 1: Univariate and Multivariate Association with Overall Survival (Surgery Only, No Chemotherapy Cohort)

\section{Figures}




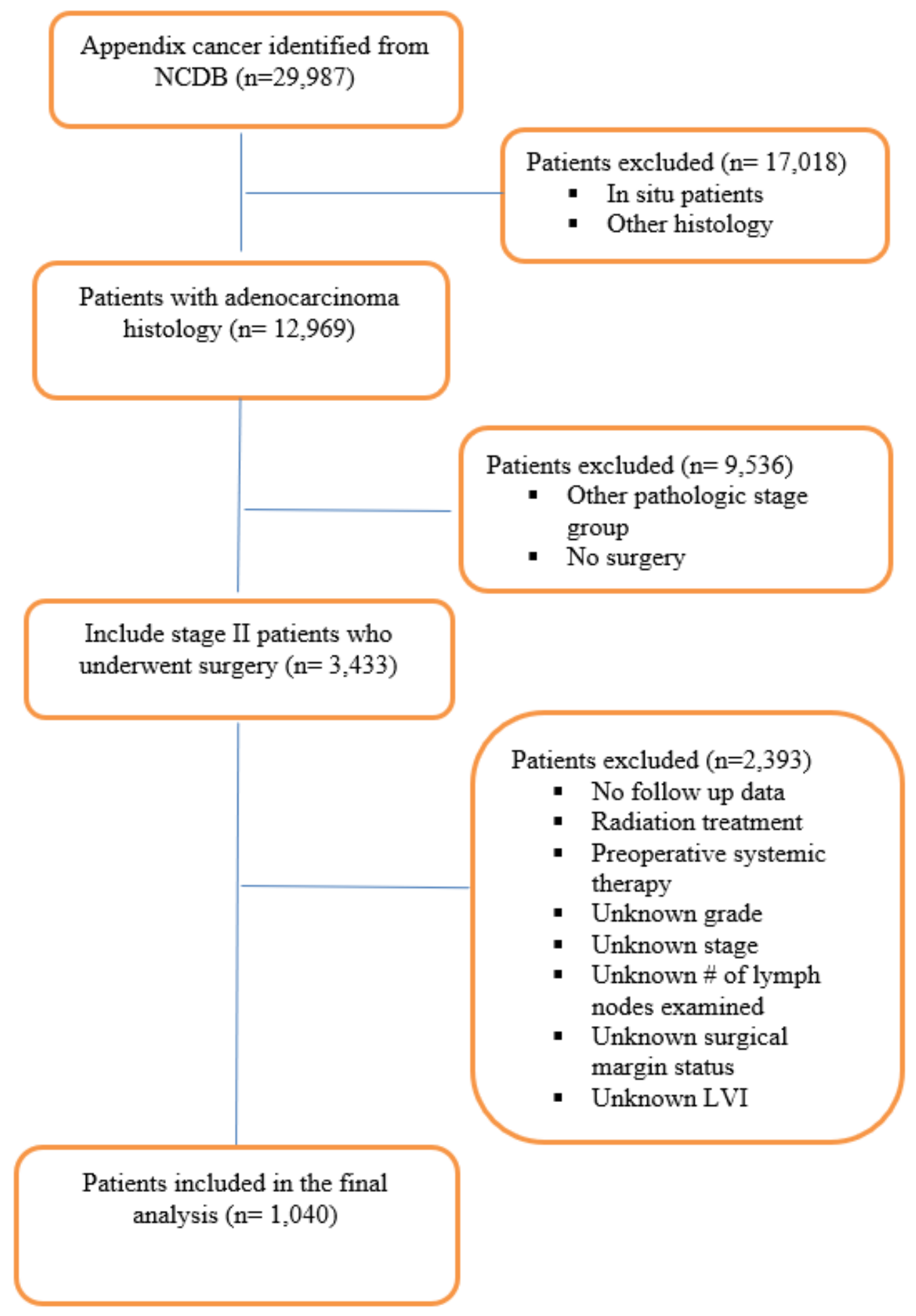

\section{Figure 1}

Consort diagram outlining the study selection 


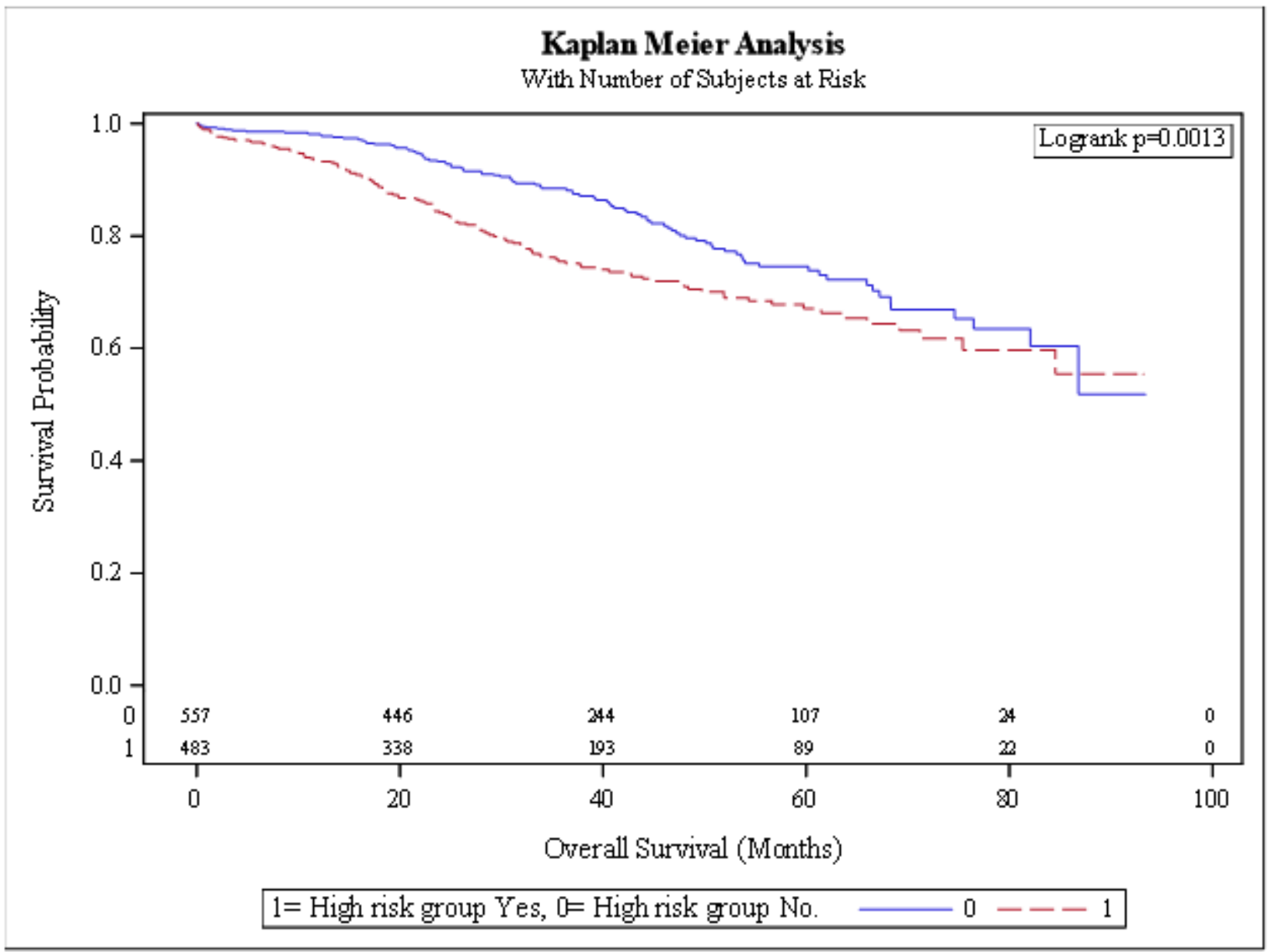

\begin{tabular}{|l|c|c|c|c|c|c|}
\hline & No. of Subject & Event & Censored & Median Survival (95\% Cl) & 12 Mo Survival & 60 Mo Survival \\
\hline Low Risk (0) & 557 & $96(17 \%)$ & $461(83 \%)$ & NA (86.8, NA) & $98.2 \%(96.6 \%, 99.0 \%)$ & $74.5 \%(69.0 \%, 79.3 \%)$ \\
\hline High Risk (1) & 483 & $122(25 \%)$ & $361(75 \%)$ & NA (84.5, NA) & $93.3 \%(90.6 \%, 95.2 \%)$ & $67.1 \%(61.4 \%, 72.1 \%)$ \\
\hline
\end{tabular}

Figure 2

Overall Survival by Risk Group (Total Cohort) 


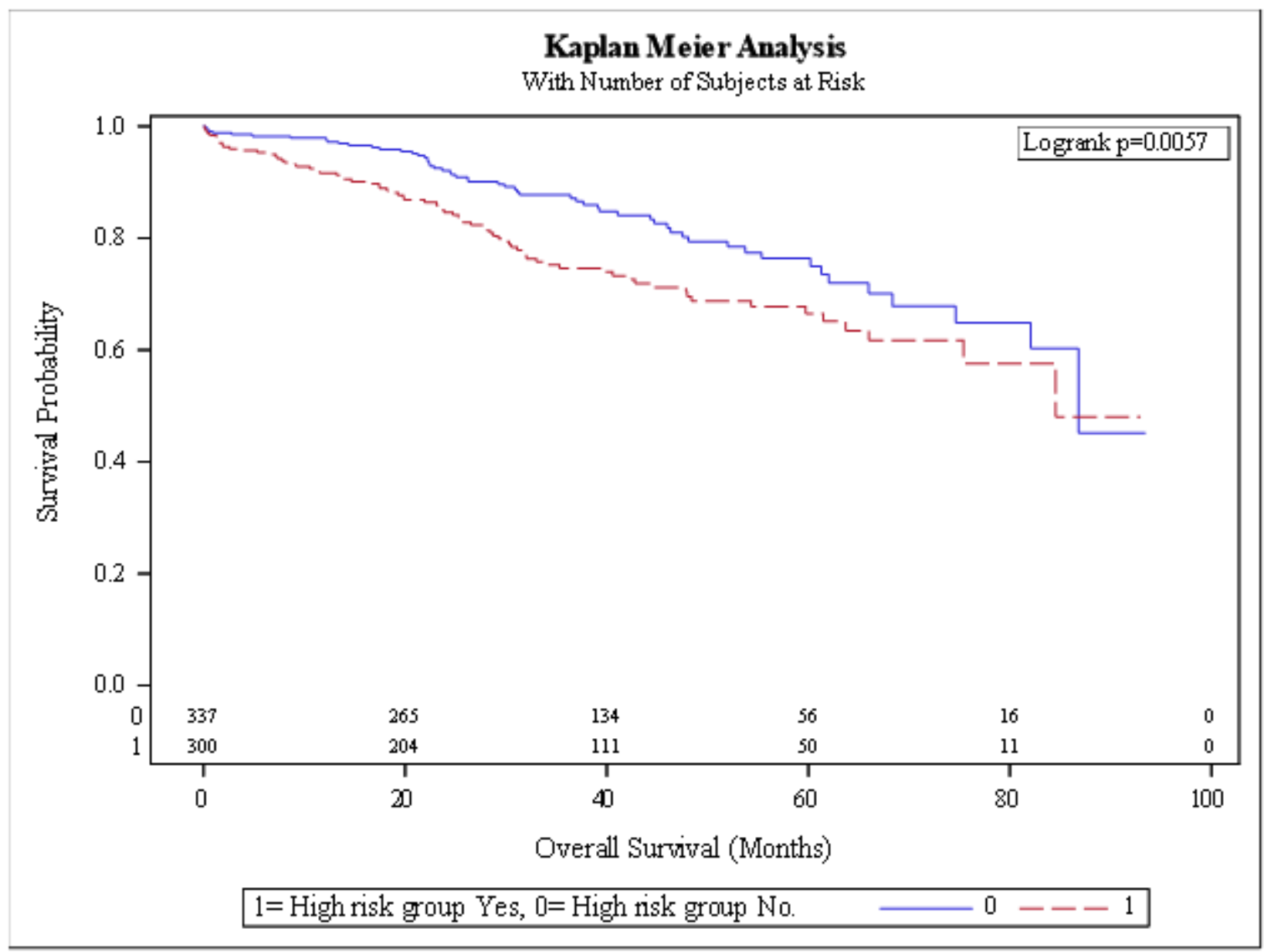

\begin{tabular}{|l|c|c|c|c|c|c|}
\hline & $\begin{array}{c}\text { No. of } \\
\text { Subject }\end{array}$ & Event & Censored & $\begin{array}{c}\text { Median Survival } \\
(95 \% \mathrm{Cl})\end{array}$ & $\mathbf{1 2}$ Mo Survival & $\mathbf{6 0}$ Mo Survival \\
\hline Low risk (0) & 337 & $56(17 \%)$ & $281(83 \%)$ & $86.8(82.1, \mathrm{NA})$ & $97.9 \%(95.6 \%, 99.0 \%)$ & $76.3 \%(69.1 \%, 82.1 \%)$ \\
\hline High risk (1) & 300 & $76(25 \%)$ & $224(75 \%)$ & $84.5(75.4, \mathrm{NA})$ & $91.6 \%(87.8 \%, 94.3 \%)$ & $66.5 \%(58.9 \%, 73.0 \%)$ \\
\hline
\end{tabular}

\section{Figure 3}

Overall Survival by Risk Group (Surgery Only, No Chemotherapy Group) 


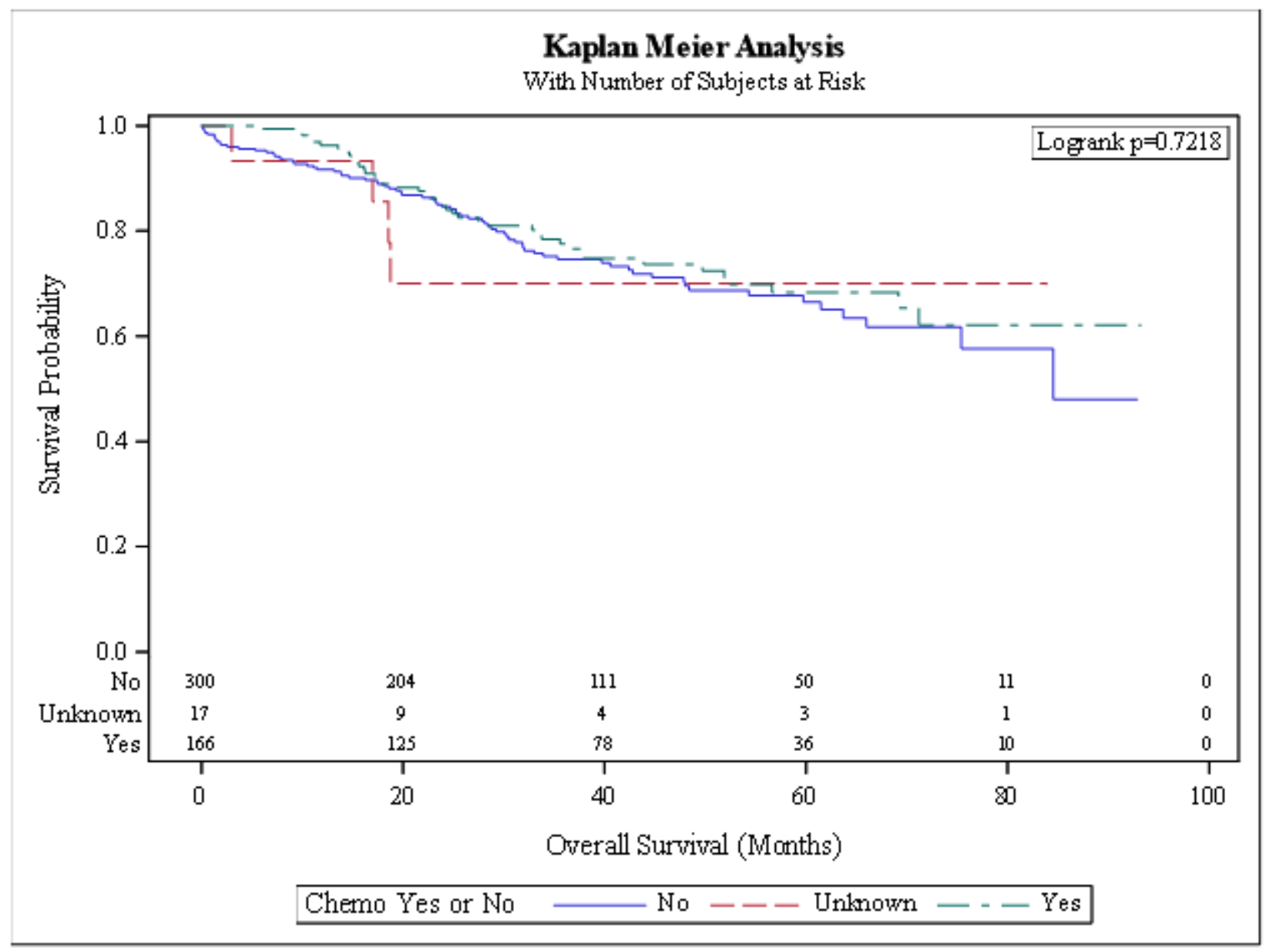

\begin{tabular}{|l|c|c|c|c|c|c|}
\hline Chemotherapy & No. of Subject & Event & Censored & Median Survival (95\% Cl) & 12 Mo Survival & 60 Mo Survival \\
\hline No & 300 & $76(25 \%)$ & $224(75 \%)$ & $84.5(75.4$, NA) & $91.6 \%(87.8 \%, 94.3 \%)$ & $66.5 \%(58.9 \%, 73.0 \%)$ \\
\hline Unknown & 17 & $4(24 \%)$ & $13(76 \%)$ & NA (18.6, NA) & $93.3 \%(61.3 \%, 99.0 \%)$ & $70.0 \%(38.2 \%, 87.6 \%)$ \\
\hline Yes & 166 & $42(25 \%)$ & $124(75 \%)$ & NA (71.2, NA) & $96.2 \%(91.8 \%, 98.3 \%)$ & $\mathbf{6 8 . 3 \% ( 5 8 . 8 \% , 7 6 . 1 \% )}$ \\
\hline
\end{tabular}

\section{Figure 4}

Overall Survival by Treatment (High Risk) 


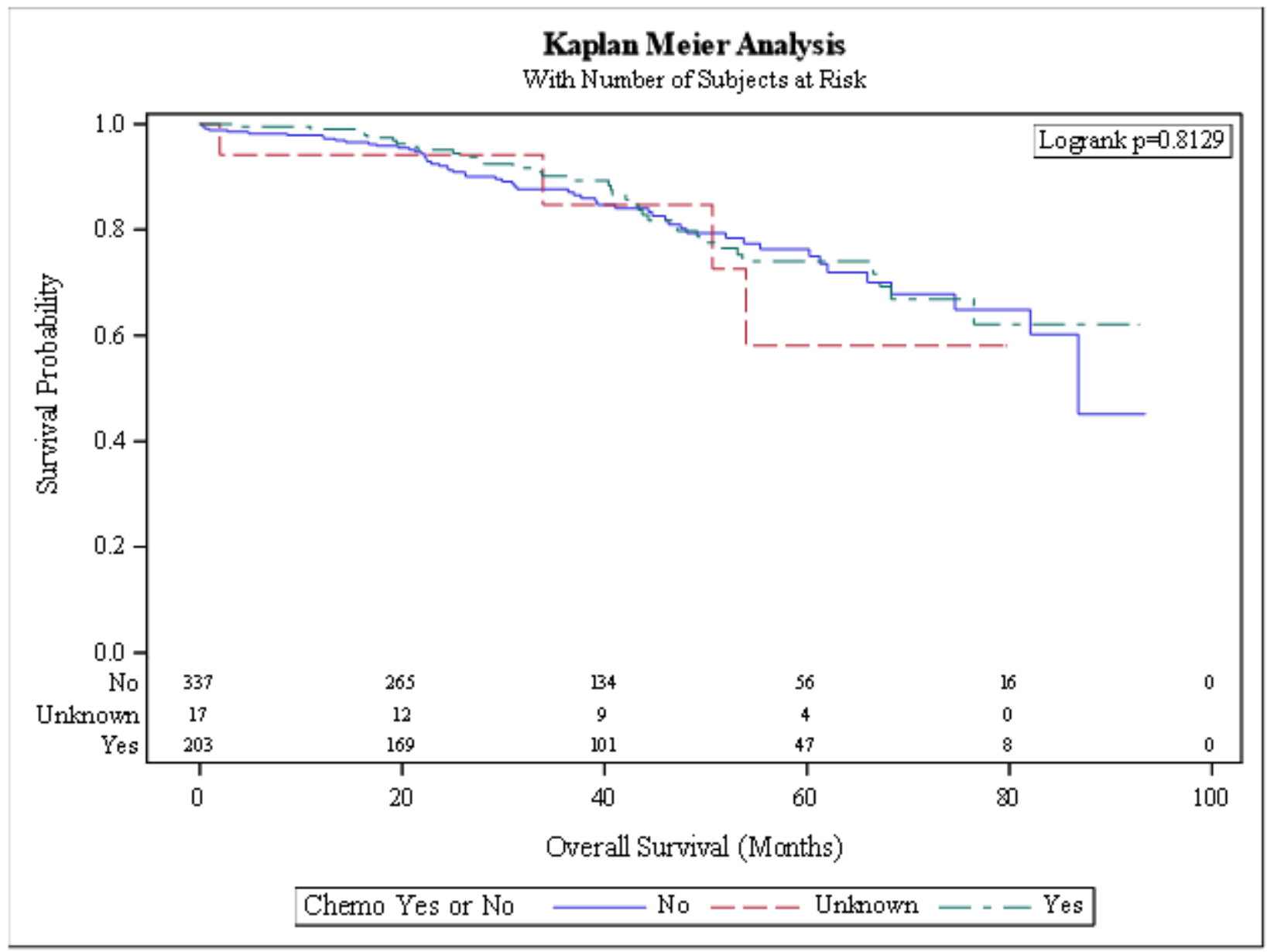

\begin{tabular}{|l|c|c|c|c|c|c|}
\hline Chemo Yes or No & No. of Subject & Event & Censored & Median Survival (95\% Cl) & 12 Mo Survival & 60 Mo Survival \\
\hline No & 337 & $56(17 \%)$ & $281(83 \%)$ & $86.8(82.1$, NA) & $97.9 \%(95.6 \%, 99.0 \%)$ & $76.3 \%(69.1 \%, 82.1 \%)$ \\
\hline Unknown & 17 & $4(24 \%)$ & $13(76 \%)$ & NA (33.9, NA) & $94.1 \%(65.0 \%, 99.1 \%)$ & $58.1 \%(20.4 \%, 83.1 \%)$ \\
\hline Yes & 203 & $36(18 \%)$ & $167(82 \%)$ & NA (76.5, NA) & $99.096(96.0 \%, 99.7 \%)$ & $74.0 \%(64.8 \%, 81.2 \%)$ \\
\hline
\end{tabular}

\section{Figure 5}

Overall Survival by Treatment (Low Risk) 